\title{
Computed tomographic evaluation of paediatric brain in a teaching hospital
}

\author{
MN Islam ${ }^{1}$, CH Rasul ${ }^{2}$, AH Sarder ${ }^{3}$, SA Hossain ${ }^{4}$
}

\begin{abstract}
During the period of June '09 to June '11, a total 689 children under 15 year, underwent brain computed tomography in the department of Radiology \& Imaging, KMCH. Trauma was the commonest chief complaint (38.6\%), followed by headache (27.3\%), convulsion (11.5\%), focal neurologic deficit (9.1\%), fever (3.5\%), developmental delay (2.9\%), increasing head size $(1.5 \%)$, and inability to straighten head $(0.9 \%)$. Traumatic events (contusion, fracture, extra axial hematoma, intra axial hematoma) were commonest CT diagnoses (24.5\%). The other diagnoses were generalized cortical atrophy (7.3\%), infarction (4.9\%), intracranial tumor (3.3 \%), congenital lesions (3.3\%), infective process (meningitis, abscess, empyema, encephalitis, tuberculoma, TORCH infection)-2.8\%, hypoxic ischemic encephalopathy (2.6\%), spontaneous intracranial hemorrhage (2.2\%), white matter disease (0.6\%). Negative finding (45\%) were also a frequent observation. Proper clinical assessment while advising pediatric brain computed tomography will reduce unnecessary CT scan and in some case initial imaging should be MRI.
\end{abstract}

\section{Introduction}

Computed tomography of brain has been performed in adults but relatively few in children. The specific uses in children are in traumatic setting, evaluation of stroke, detecting atrophic conditions, identifying intracranial mass lesions and the character of the lesion, and evaluating congenital cranial diseases such as cerebral dysplasia, tuberous sclerosis and leukodystrophies.' This is safe, rapid, noninvasive technique, so the indication of pediatric brain imaging are increasing day by day.

Imaging of the pediatric brain is challenging. Knowledge of the normal anatomy and appearance of the developing brain at a given age is critical in identifying abnormalities.2 Hightech computed tomography today provides good anatomic depiction of the brain and its gross disease processes that have a manifestation as alteration of brain density, disturbance in blood-brain barrier or mass effect. As such, computed tomography often provides valuable diagnostic information in the appropriate clinical setting. 3

In the Radiology \& Imaging department of Khulna medical college hospital (KMCH), computed tomography was started from June 2009 by a 16 slice scanner which able to scan faster as well as more efficacious in the detection of lesion conspiciously. The patient demography, clinical indication \& computed tomographic findings should have to be analyzed to elucidate relative frequency of computed tomographic diagnoses in relation to symptoms, cross tabulation of symptom \& findings would show the relative strength \& weakness of computed tomography in particular clinical setting or symptom. Interpretations of this knowledge should obviously further enhance diagnostic yield of computed tomography so that the clinician as well as radiologist will be beneficiary. Current study aims to analyze retrospective data to see relative frequency of pediatric brain computed tomographic diagnoses and their cross tabulation to patients chief complaints. No such study has been noticed so far, so current series will set a reference value for the evaluation of pediatric brain by computed tomography.

Patients and methods

Retrospective cross-sectional study is performed in Khulna medical college hospital, a tertiary level regional referral health facility \& teaching hospital, at Radiology \& Imaging
Bang Med J (Khulna) 2011; 44 : 3-6

department. During the period June '09 to June '11, patient under 15 year age group, a total 689 pediatric brain scanning done, patient were referred from outpatient \& inpatient departments. CT scanning was done by 16 slice scanner, records of patients aged upto 15 years over a period of June ' 09 to June ' I 1 were analyzed for chief complaints of the examination \& CT diagnosis. Imaging finding/ Computed Tomographic diagnoses were grouped as congenital, traumatic, infarction, spontaneous intracerebral hemorrhage, Intra cranial space occupying lesion (ICSOL), Generalized cortical atrophy (GCA), infection (meningitis, cerebral abscess, empyema, , encephalitis, TORCH), white matter disease and others. The congenital, ICSOL \& infection diagnoses were further sub grouped into particular clinical entities. The diagnoses were cross tabulated against there chief complaints \& age distribution. Data were analyzed by SPSS version 14.0 for Windows and presented in tables and pie charts.

Result

Chief complaints

From June '09 to June ' I I a total 689 cases of 5 days-15 years' pediatric CT performed. Among them Trauma were the commonest chief complaint (38.6\%) that necessitated the request for CT scan, followed by headache (27.3\%), and convulsion (11.5 $\%)$, focal neurologic deficit (9.1\%), fever (3.5\%), developmental delay (2.9\%), increasing head size (1.5\%), inability to straighten head $(0.9 \%)$, other complaint comprises $3.9 \%$ which includes vomiting, generalized weakness, disfigurement of skull, restlessness etc.

CT diagnosis

Traumatic events (contusion, fracture, extra axial hematoma, intra axial hematoma) were commonest positive findings (24.5\%). Next in frequency were

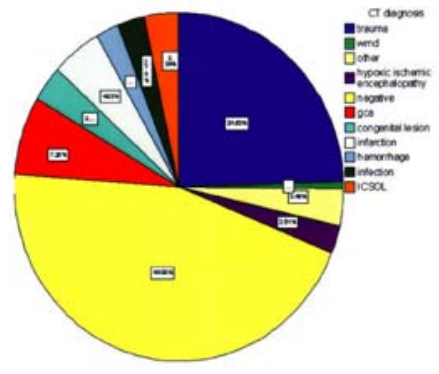

Fig. I : Computed tomographic diagnoses

1. Mohammad Nazrul Islam MD. Assistant Professor of Radiology, Khulna Medical College, Khulna.

2. Choudhury Habibur Rasul FCPS, Professor of Paediatrics, Khulna Medical College, Khulna.

3. Abdul Halim MD. Assistant Professor of Neuro Medicine, Khulna Medical College, Khulna.

4. Shaikh Amir Hossain FCPS, Assistant Professor of Medicine, Khulna Medical College, Khulna. 
generalized cortical atrophy (GCA-7.3\%), infarction (4.9\%) and intracranial tumor (3.3\%), (Figure-1). Other diagnoses were $3.5 \%$ which comprises hemimegalencephaly, diffuse brain edema, sub dural hygroma, communicating hydrocephalus etc.

Table I

Age group wise CT diagnoses

$\begin{array}{llllllllllll}\begin{array}{l}\text { age } \\ \text { group }\end{array} & \begin{array}{l}\text { trau- } \\ \text { ma }\end{array} & & \text { WMD } & \text { other } & \text { HIE } & \begin{array}{l}\text { nega- } \\ \text { tive }\end{array} & \begin{array}{l}\text { GCA } \\ \text { con- } \\ \text { geni }\end{array} & \begin{array}{l}\text { in- } \\ \text { farc }\end{array} & \begin{array}{l}\text { hemo- } \\ \text { rrhage }\end{array} & \begin{array}{l}\text { in- } \\ \text { farc }\end{array} \text { OL } \\ \text { Unter } 1 & 7 & 0 & 3 & 11 & 4 & 18 & 10 & 2 & 1 & 5 & 0 \\ 2-5 & 38 & 1 & 4 & 3 & 30 & 12 & 3 & 13 & 4 & 3 & 6 \\ 6-10 & 76 & 2 & 10 & 3 & 100 & 10 & 5 & 11 & 6 & 7 & 12 \\ 11-15 & 48 & 1 & 7 & 1 & 176 & 10 & 5 & 8 & 4 & 4 & 5 \\ \text { Total } & 169 & 4 & 24 & 18 & 310 & 50 & 23 & 34 & 15 & 19 & 23\end{array}$

Age group \& CT diagnosis cross tabulation

Most of the congenital lesion, hypoxic ischemic encephalopathy, generalized cortical atrophy were occurring below 1 year group. ICSOL, infarction \& hemorrhage were more prevalent at 6-10 year group. (Table-1)

CT diagnosis of congenital lesion

Out of 689 cases, congenital lesion was in 23 cases, among them commonest congenital lesion

Table II

Frequency of congenital lesions

percent

Aqueductal stenosis

Frequency Relative

Myelomeningocele

30.4

Dandy walker malformation

21.7

Tuberous sclerosis

17.4

Sterge weber syndrom

8.6

8.6

Corpus callosal agenesis

8.6

Liscencephaly

4.3

Total

100.00

was acqueductal stenosis (7 cases) followed by myelomenigocele (5 cases), Dandy Walker malformation (4 cases), Tuberous sclerosis (2 cases), Sterge-Weber syndrome (2 cases) and corpus callosal agenesis (2 cases) (Table-I1)

Table V

Correlation of symptoms to findings

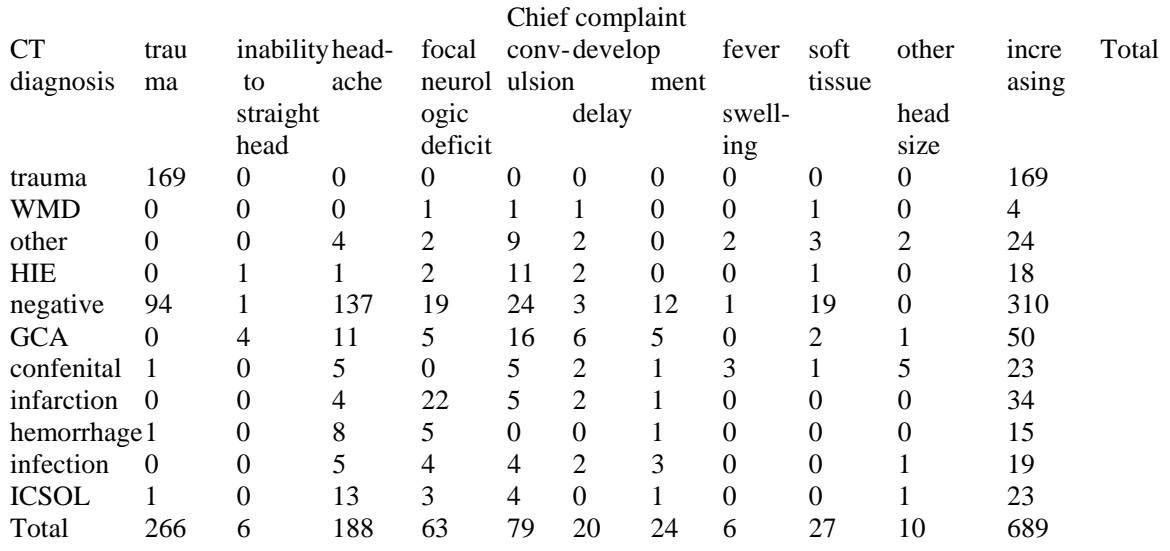

Table III

Frequency of ICSOL

$\begin{array}{lll} & \text { Frequency } & \text { Relative percent } \\ \text { extra axial } & 8 & 34.8 \\ \text { supratentorial } & 7 & 30.4 \\ \text { infratentorial } & 5 & 21.8 \\ \text { intraventricular cyst } & 3 & 13 \\ \text { Total } & 23 & 100\end{array}$

CT infection pattern

19 cases were infective in nature, meningitis is commonest CNS infection (7 cases) followed by TORCH infection (5cases), cerebral abscess (3 cases), encephalitis (2 cases), sub dural empyema(1 case) $\&$ tuberculoma (1 case)- (Table-IV).

Table IV

Frequency of infection pattern

$\begin{array}{lll} & \text { Frequency } & \text { Percent } \\ \text { Meningits } & 7 & 1.0 \\ \text { Torch infection } & 5 & 0.7 \\ \text { Cerebral abscess } & 3 & 0.4 \\ \text { Encephalitis } & 2 & 0.3 \\ \text { Tuberculoma } & 1 & 0.1 \\ \text { Sub dural empyema } & 1 & 0.1 \\ \text { Total } & 19 & 2.5\end{array}$

CT diagnosis \& chief complaint

10 cases complained increasing head size, all of them (100\%) shows positive findings. 20 patients complaints of developmental delay, among them 17 cases (85\%) showed CT Positive finding. Inability to straighten head was chief complaints of 6 cases and 5 (83\%) cases showed positive findings, 63 cases presented as focal neurologic deficit and on CT examination 44 cases(69\%) yielded positive CT findings, convulsion was complaints of 79 cases and 55 cases (69\%)shows positive findings, complaints of having trauma was in 266 cases and positive findings were in 172 cases (64\%). Overall positive finding was 55\%. Negative findings are more in chief complaints of headache (73\%), trauma (36\%), focal neurological deficit (31\%) and in convulsion (31 \%)-(Table-V). 


\section{Discussion}

Trauma is the leading cause of death and disability in children.4,5 Our series showed trauma as commonest chief complaint (38.6 \%) that necessitated the request for CT scan and traumatic finding as commonest positive findings (24.5 $\%)$. Adolescents \& toddlers were affected more by traumatic injury during their play \& lack of their self protection.

Headache was lowest positive finding yielding (27\%) complaint; though we have detected more positive findings in headache than other series like Deda et all (12\%)6, Nawaz et all (4\%)7 \& Lateef et all (4\%)8. Thus Maytal concluded that brain imaging studies have limited value in evaluating headaches in pediatric patients without clinical evidence of an underlying structural lesion.9 In our series the more positive finding in headache might be due to our 16 slice scanner which has increased lesions conspicuously. Top positive finding yielding complaints were increasing head size $(100 \%)$, developmental delay $(85 \%)$, inability to straighten head (83\%), focal neurologic deficit (69\%), convulsion (69\%), trauma (64\%) making CT scan as imaging study of choice in these complaints. Overall positive finding rate (55 \%) is higher than other study possibly due to increased lesion detection by 16 slice scanner in our center. We can further increase the rate by careful selection of cases by clinical evaluation in headache, focal neurological deficit or convulsion, thus we can avoid unnecessary CT scanning in pediatric patients. In this regard clinician may consider other modality such as MRI to evaluate such complaints.

Brain malformations represented important factor of morbidity and mortality in children. Our series showed that most predominant malformation was acqueductal stenosis or congenital triventricular hydrocephalus (30.4\%) followed by neural tube defect or myelomenigocele (21.7\%), Dandy Walker malformation (17.4\%), tuberous sclerosis (8.6\%), Sterge-Weber syndrome (8.6\%) and corpus callosal agenesis (8.6\%). These findings had close similarity to series of Hadzagic et al finding of neural tube defects $(38,6 \%)$, hydrocephalus $(26,8 \%)$, agenesis of corpus callosum in $(7,9 \%)$, Dandy Walker malformation in $(4,7 \%)$ .10

Brain tumors are categorized by site into two groups, supratentorial and infratentorial. In a series of 44 cases by Haque et all $42.9 \%$ cases the tumors were supratentorial and in $57.1 \%$, they were infratentorial in location.11 Naseem et al shows these locations as $33.3 \%$ \& $66.7 \% .12$ Our study shows supratentorial tumors were slightly more than infratentorial which are also seen in the series of Rickert et al as supratentorial locations predominated $53.3 \% .13$ In children, the recognition of stroke is often delayed due to the low incidence of stroke and the lack of specific assessment measures to this entity \& the causes of pediatric stroke are significantly different from that of adult stroke.14 Global hypoxic-ischemic encephalopathy/injury (HIE) to the brain is a significant cause of mortality and severe neurologic disability. Hypoxic-ischemic injury \& generalized cortical atrophy (GCA) indicate perinatal \& neonatal ischemic insult.15

Our series shows CT diagnoses of HIE \& GCA comprises $9.87 \%$ of diagnoses, most were associated with the complaints of convulsion, developmental delay \& focal neurologic deficit. CT diagnoses of meningitis, tuberculoma \& encephalitis was only $1 \%, 0.1 \%$ \& $0.3 \%$, these lower detection rate could be due to lower efficacy of computed tomography in these settings, so patient suspected to have such disease should be advised for contrast enhanced MRI, similar observation made by Inderjeet et al as more patients with meningitis are undergoing CT and the number of inappropriate requests are increasing.16 In cases of uncomplicated meningitis, cranial computed tomography (CT) appears to be sufficient for clinical management to exclude acute brain edema, hydrocephalus, and pathology of the base of skull. Magnetic resonance imaging (MRI) is superior in depicting complications like sub-/epidural empyema and vasculitic complications notably on FLAIR (fluid-attenuated inversion recovery) weighted images. The newer technique of diffusion-weighted imaging (DWI) shows early parenchymal complications of meningitis earlier and with more clarity and is of help in differentiation of pyogenic abscess from ring enhancing lesions of other etiology.17

Conclusion

Computed tomography will enhance and expand the practice of radiology for benefit of the sick child particularly with the complaints of increasing head size, developmental delay, inability to hold head upright, focal neurological deficit, convulsion and trauma, they are also helpful in evaluation of headache \& fever.

References

1. Harwood-Nash DC, Charles R, Bernard J. Cranial computed tomography in infants and children. CMA JOURNAL. 1975; 20:113.

2. Ketonen LM, Valanne L. Neuroimaging of pediatric diseases. Semin Neurol. 2008; 28:558-69. Epub 2008.

3. Hershey BL, Zimmerman RA Pediatric brain computed tomography. Pediatr Clin North Am. 1985;32:1477-508.

4. Pediatric Head Trauma: Parent, Parent-Child and Family Functioning 2 Weeks after Hospital Discharge. J Pediatr Psychol. 2006; 31: 608-618.

5. Mary Hartman, Robert Scott Watson, Walter Linde-Zwirble, Gilles Clermont, Judith Lave, Lisa Weissfeld, Patrick Kochanek and Derek Angus.Pediatric Traumatic Brain Injury Is Inconsistently Regionalized in the United States. Pediatrics 2008; 122: e172-e180

6. Deda G, Caksen H, Ocal A. Headache etiology in children: a retrospective study of 125 cases. Pediatr Int. 2000;42:668-73.

7. Nawaz M, Amin A, Qureshi AN, Jehanzeb M. Audit of appropriateness and outcome of computed tomography brain scanning for headaches in paediatric age group. J Ayub Med Coll Abbottabad. 2009; 21: 91-3.

8. Lateef TM, Grewal M, McClintock W, Chamberlain J, Kaulas H, Nelson KB. Headache in young children in the emergency department: use of computed tomography. Pediatrics. 2009; 124: e12-7.

9. Maytal J, Bienkowski RS, Patel M, Eviatar L.The value of brain imaging in children with headaches. Pediatrics. 1995; 96: 413-6.

10. Hadzagic-Catibusic F, Maksic H, Uzicanin S, Heljic S, Zubcevic S, Merhemic Z, Cengic A, Kulenovic E.Congenital malformations of the central nervous system: clinical approach. Bosn J Basic Med Sci. 2008; 356-60.

11. Haque MZ, Karim ME, Al-Azad S, Mahmood-uz jahan. Role of computed tomography in the evaluation of pediatric brain tumor.Bangladesh Med Res Counc Bull. 2010; 36: 89-92.

12. Naseem Ahmedl, Yasmin Bhurgri, Saleem Sadiql, Kazi A. Pediatric Brain Tumours at a Tertiary Care Hospital in Karachi Shakoor.Asian Pacific J Cancer Prev, 2007; 8: 399-404

13. Rickert $\mathrm{CH}$, Paulus W. Epidemiology of central nervous system tumors in childhood and adolescence based on the new WHO classification. Childs Nerv Syst.2001; 17: 503-11.

14. Hueng-Chuen Fan, Chih-Fen Hu, Chun-Jung Juan, and Shyi-Jou Chen. Current Proceedings of Childhood Stroke. Stroke Res Treat. 2011; 43 : 283-9.

15. Benjamin Y. Huang, Mauricio Castillo. Hypoxic-Ischemic Brain Injury: Imaging Findings from Birth to Adulthood. RadioGraphics, 2008; 28, 417-439.

16. Inderjeet Nagra, Bernard Wee, Jennifer Short, Arpan K Banerjee. The role of cranial CT in the investigation of meningitis. JRSM Short Rep. 2011; 2: 20.

17. Oliver Kastrup, Isabel Wanke, Matthias Maschke. Neuroimaging of Infections. NeuroRx. 2005;2:324-332 